\title{
HOMOLOGICALLY THIN, NON-QUASI-ALTERNATING LINKS
}

\author{
JoshuA Greene
}

\begin{abstract}
We exhibit the first examples of links which are homologically thin but not quasi-alternating. To show that they are not quasi-alternating, we argue that none of their branched double-covers bounds a negative definite 4-manifold with non-torsion $H_{1}$. Using this method, we also complete the determination of the quasi-alternating pretzel links.
\end{abstract}

\section{Introduction.}

Quasi-alternating links were defined by Ozsváth and Szabó [31, Definition 3.9]. They are a natural generalization of the class of alternating links.

Definition 1.1. The set $\mathcal{Q}$ of quasi-alternating (QA) links is the smallest set of links such that

- the unknot $U$ belongs to $\mathcal{Q}$, and

- if $L$ is a link with a projection containing a crossing for which the two resolutions $L_{0}$ and $L_{1}$ belong to $\mathcal{Q}$, and $\operatorname{det}(L)=\operatorname{det}\left(L_{0}\right)+\operatorname{det}\left(L_{1}\right)$, then $L$ belongs to $\mathcal{Q}$.

By this definition, non-split alternating links belong to $\mathcal{Q}$. QA links arise naturally in connection with various link homology theories. The relevant invariants here are versions of the knot Floer and Khovanov homology groups, and take the form of a bigraded, finitely-generated abelian group. A link is thin in one of these theories if its homology is supported on a single diagonal with respect to the bigrading.

The following properties hold for a QA link $L$ :

(1) the knot Floer homology group $\widehat{H F K}(L ; \mathbb{Z} / 2 \mathbb{Z})$ is thin [21, Theorem 2];

(2) the reduced ordinary Khovanov homology group $\overline{K h}(L ; \mathbb{Z})$ is thin and torsionfree [21, Theorem 1];

(3) the reduced odd Khovanov homology group $\overline{K h}^{\prime}(L ; \mathbb{Z})$ is thin and torsion-free [26, remark after Proposition 5.2]; and

(4) the branched double-cover $\Sigma(L)$ is a Heegaard Floer L-space, and it bounds a negative definite 4-manifold $W$ with $H_{1}(W)=0$ [31, Proposition 3.3 and the proof of Lemma 3.6].

It is an interesting open problem to characterize those links that are thin with respect to any one of the above link homology theories. For some time, it remained a possibility that a link was $\widehat{H F K}$ - or $\overline{K h}$-thin if and only if it was QA. This possibility was recently refuted by Shumakovitch, who used his excellent program KhoHo [33]

Received by the editors June 11, 2009.

Partially supported by an NSF Post-doctoral Fellowship. 


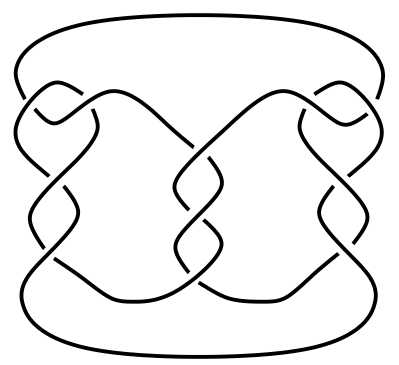

Figure 1. The knot $11 n 50$.

to show that the pretzel knots $9_{46}=P(3,-3,3)$ and $10_{140}=P(3,-3,4)$ have (offdiagonal) torsion in their odd Khovanov homology groups, although they are both thin and torsion-free with respect to $\widehat{H F K}$ and $\overline{K h}$. Thus, neither of these knots is odd-thin, so neither is QA. ${ }^{1}$

Definition 1.2. A link $L$ is homologically thin (without qualification) if it is simultaneously thin and torsion-free with respect to $\widehat{H F K}, \overline{K h}$, and $\overline{K h}^{\prime}$.

It has remained an open problem to exhibit a link that is homologically thin and not QA. The purpose of this note is to describe such examples, and moreover to exploit the second part of condition (4) as an obstruction to QA-ness.

Theorem 1.3. There exist homologically thin, non-QA links.

At the heart of our approach is Donaldson's celebrated "Theorem A", which asserts that the intersection pairing of a smooth, closed, negative definite 4-manifold is diagonalizable [4]. Coupled with calculations by several researchers $[1,3,11,20,33,36]$, we identify $11 n 50$ as the only knot with up to 11 crossings which is neither QA nor odd-thick. Furthermore, combined with work of Champanerkar-Kofman [3, Theorem 3.2 , we complete the determination of the QA pretzel links. For a clear, concise account of the relevant notation and facts concerning Montesinos links here and in what follows, see [24, Section 3.2].

Theorem 1.4. The pretzel link $P\left(-e ; p_{1}, \ldots, p_{n},-q_{1}, \ldots,-q_{m}\right)=M\left(-e ;\left(p_{1}, 1\right), \ldots\right.$, $\left.\left(p_{n}, 1\right),\left(q_{1},-1\right), \cdots,\left(q_{m},-1\right)\right)$, with $e, n, m \geq 0$, all $p_{i} \geq 2$, and all $q_{j} \geq 3$, is QA iff

(1) $e>m-1$;

(2) $e=m-1>0$;

(3) $e=0, n=1$, and $p_{1}>\min \left\{q_{1}, \ldots, q_{m}\right\}$ or $m \leq 1$; or

(4) $e=0, m=1$, and $q_{1}>\min \left\{p_{1}, \ldots, p_{n}\right\}$ or $n \leq 1$.

The same is true on permuting the parameters $p_{i}$ and $q_{j}$.

Any pretzel link can be put in the above form after mirroring [13, Section 2.3], which clearly preserves the QA property. For comparison, a pretzel link in the above notation is alternating iff $e>m-1, e=n=0$, or $m+n \leq 2$. For if $m+n \leq 2$,

\footnotetext{
${ }^{1}$ In fact, in all known examples, an odd-thin link is $\overline{K h}$-thin, and a link is $\overline{K h}$-thin iff it is $\widehat{H F K}$ thin. A conjecture of Rasmussen would imply that a $\overline{K h}$-thin link is necessarily $\widehat{H F K}$-thin $[32$, Section 5].
} 


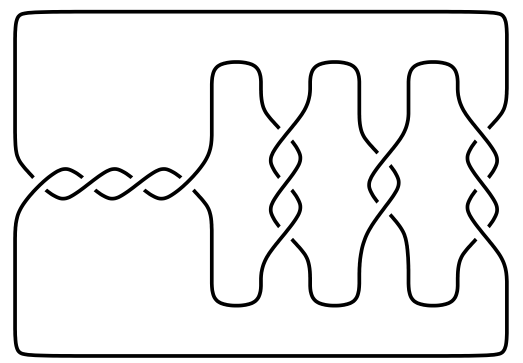

Figure 2. The pretzel link $P(-4 ; 3,2,-3)$.

then the link is 2-bridge, hence alternating. Otherwise, it has a reduced Montesinos diagram and its crossing number is attained by this diagram [19, pp. 535-536 and Theorem 10]. Therefore, the link is alternating iff its reduced Montesinos diagram is [18, Corollary 5.10], which occurs iff $e>m-1$ or $e=n=0$.

Section 2 contains the proofs of Theorems 1.3 and 1.4. Section 3 contains some related examples, and sketches the construction of an infinite family of homologically thin, non-QA hyperbolic knots [8]. It concludes with a discussion surrounding Conjecture 3.1, which asserts that there are finitely many QA links of bounded determinant.

\section{Proofs of the main results.}

To prove the theorems, we rely on the following lemma. We use (co)homology groups with integer coefficients throughout.

Lemma 2.1. Suppose that $X$ and $W$ are a pair of 4-manifolds, $\partial X=-\partial W=Y$ is a rational homology sphere, and $H_{1}(W)$ is torsion-free. Express the map $H_{2}(X) /$ Tors $\rightarrow$ $H_{2}(X \cup W) /$ Tors with respect to a pair of bases by the matrix A. This map is an inclusion, and if some $k$ rows of $A$ contain all the non-zero entries of some $k$ of its columns, then the induced $k \times k$ minor has determinant \pm 1 .

Proof. The stated assumption ensures that the restriction map $H^{2}(X \cup W) \rightarrow H^{2}(X)$ surjects. Just to be sure, consider the long exact sequences in cohomology for the pairs $(X \cup W, X)$ and $(W, Y)$, and the natural map between them. The relevant portion reads

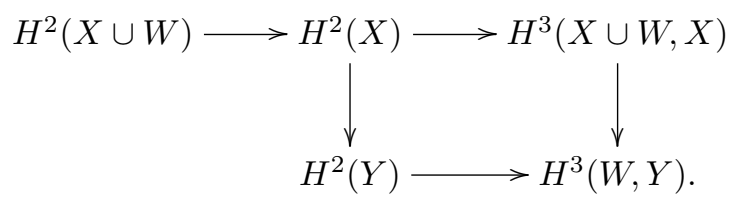

The second vertical arrow is an isomorphism by excision, and Poincaré-Lefschetz duality identifies this group with $H_{1}(W)$, which is torsion-free. Since $H^{2}(Y)$ is torsion, the bottom horizontal map is 0 . It follows that the map $H^{2}(X) \rightarrow H^{3}(X \cup W, X)$ is 0 , so the map $H^{2}(X \cup W) \rightarrow H^{2}(X)$ surjects as claimed. 


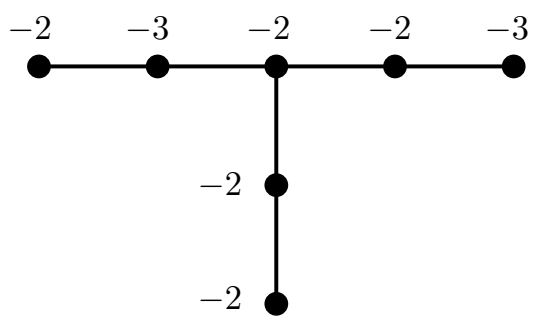

FiguRE 3. The graph $\Gamma$.

Consequently, the map $H^{2}(X \cup W) /$ Tors $\rightarrow H^{2}(X) /$ Tors surjects as well. On the other hand, this map of groups is dual to the map $H_{2}(X) /$ Tors $\rightarrow H_{2}(X \cup W) /$ Tors, so is given with respect to the pair of dual bases by the matrix $A^{T}$. Suppose that some $k$ rows of $A$ contain all the non-zero entries of some $k$ of its columns, and let $B$ denote the corresponding $k \times k$ minor. By permuting the basis elements if necessary, we may assume that $B$ is the top-left $k \times k$ minor, possibly changing its determinant by a sign:

$$
A=\left(\begin{array}{cc}
B & C \\
0 & D
\end{array}\right)
$$

Since the dual map $A^{T}$ surjects, the map $B^{T}$ must as well, hence $\operatorname{det}(B)= \pm 1$, as claimed. The fact that the map $A$ injects follows, for example, from the Mayer-Vietoris sequence for the natural decomposition of $X \cup W$, noting that $H_{2}(Y)$ vanishes.

Proof of Theorem 1.3. We establish the result by showing that $K=11 n 50$ is homologically thin but not QA. Additional examples appear in Subsection 3.2. The knot Floer homology group $\widehat{H F K}(K ; \mathbb{Z} / 2 \mathbb{Z})$ was calculated by Baldwin-Gillam [2], and the Khovanov homology groups $\overline{K h}(K)$ and $\overline{K h}^{\prime}(K)$ by Shumakovitch [33]; their results show that $K$ is homologically thin.

Figure 1 exhibits the knot $11 n 50$ as the Montesinos knot $M(0 ;(5,2),(3,1),(5,-2))$, which is equivalent to $M(1 ;(5,2),(3,1),(5,3))$. As such, its branched double-cover $Y$ is the boundary of the plumbing $X$ on the graph $\Gamma$ shown in Figure 3 (cf. [24, Section 3.2]). We label the vertices of $\Gamma$ from left to right and top to bottom by $v_{1}, \ldots, v_{7}$. The spheres corresponding to these vertices give rise to a basis for $H_{2}(X)$, with respect to which the intersection pairing on $X$ is given by the weighted adjacency matrix $A_{\Gamma}$, whose $(i, i)$-entry records the weight of $v_{i}$, and whose $(i, j)$-entry for $i \neq j$ is 1 or 0 according as $v_{i}$ and $v_{j}$ are adjacent or not. The space $X$ is negative definite and $H_{1}(X)=0$.

If $11 n 50$ were $\mathrm{QA}$, then its mirror $\overline{11 n 50}$ would be as well, whose branched doublecover is $-Y$. We proceed by way of fact (2) to derive a contradiction. According to it, there must exist a negative definite 4-manifold $W$ with $\partial W=-Y$ and $H_{1}(W)=0$. Consider the 4-manifold $X \cup W$. It is a closed, smooth, negative definite 4-manifold, so by Donaldson's theorem, its intersection pairing is diagonalizable. That is, there exists an isomorphism $\left(H_{2}(X \cup W) /\right.$ Tors, $\left.Q_{X \cup W}\right) \cong-\mathbb{Z}^{n}$, where $n=b_{2}(X \cup W)$ and 
$-\mathbb{Z}^{n}=\left\langle E_{1}, \ldots, E_{n}\right\rangle$ denotes the space $\mathbb{Z}^{n}$ equipped with minus its standard positive definite inner product.

Therefore, $\left(H_{2}(X) /\right.$ Tors, $\left.Q_{X}\right)$ embeds into $-\mathbb{Z}^{n}$, for some $n$. Let $x_{i}$ denote the image of the class in $H_{2}(X) /$ Tors under the inclusion into $-\mathbb{Z}^{n}$ which corresponds to the vertex $v_{i}$. No pair of vertices of weight -2 has the same set of neighbors, so the corresponding vectors in $-\mathbb{Z}^{n}$ have distinct reductions $(\bmod 2)$. This observation helps us to deduce that, by applying an automorphism to $-\mathbb{Z}^{n}$, we have $x_{7}=E_{1}-$ $E_{2}, x_{6}=E_{2}-E_{3}, x_{3}=E_{3}-E_{4}, x_{4}=E_{4}-E_{5}$, and $x_{1}=E_{6}-E_{7}$. Now, swapping $E_{6}$ and $-E_{7}$ if need be, we obtain $x_{2}=E_{4}+E_{5}-E_{6}$, and then $x_{5}=E_{5}+E_{6}+E_{7}$. Thus, with respect to the chosen bases for $H_{2}(X) /$ Tors and $H_{2}(X \cup W) /$ Tors, the inclusion map is given by a matrix $A$ whose seven columns are supported in its first seven rows. Let $B$ denote the induced $7 \times 7$ minor. Then $-B^{T} B=-A^{T} A=A_{\Gamma}$, and this is a presentation matrix for $H^{2}(Y) \cong \mathbb{Z} / 25 \mathbb{Z}$. Hence $|\operatorname{det}(B)|=5 \neq 1$, in contradiction to Lemma 2.1.

It follows that $-Y$ does not bound a negative definite 4-manifold with torsion-free, let alone vanishing, $H_{1}$, and so the knot $11 n 50$ is not QA.

We remark that there does exist a negative definite 4-manifold $W$ with boundary $-Y$ for which $H_{1}(W)$ contains torsion. The knot $11 n 50$ is a slice $k_{n o t}^{2}$, so the doublecover of $D^{4}$, branched along a slice disk for the mirror $\overline{11 n 50}$, is a rational homology ball with boundary $-Y$, which we may blow up to make undeniably negative definite. However, its first homology group contains a subgroup isomorphic to a quotient of $H_{1}(Y)$ of square-root order, which is $\mathbb{Z} / 5 \mathbb{Z}$ in this case.

Proposition 2.2. For $n \geq 2, p_{1}, \ldots, p_{n} \geq 2$, and $q \geq 1$, the pretzel link $P\left(0 ; p_{1}, \ldots\right.$, $\left.p_{n},-q\right)$ is $Q A$ iff $q>\min \left\{p_{1}, \ldots, p_{n}\right\}$.

Proof. Let $L$ denote the pretzel link $P\left(0 ; p_{1}, \ldots, p_{n},-q\right)=M\left(0 ;\left(p_{1}, 1\right), \ldots,\left(p_{n}, 1\right)\right.$, $(q,-1))=M\left(1 ;\left(p_{1}, 1\right), \ldots,\left(p_{n}, 1\right),(q, q-1)\right)$. The space $\Sigma(L)$ is the boundary of the plumbing $X$ on a star-shaped planar graph $\Gamma$. The graph $\Gamma$ has $n+1$ rays of lengths $p_{1}-1, \ldots, p_{n}-1$, and 1 emanating from the star vertex in cyclic order; by length we mean the number of edges. The star vertex receives weight $-n$, the vertex on the distinguished ray of length 1 receives weight $-q$, and every other vertex receives weight -2 . As before, the intersection pairing on $X$ is given in the natural spherical basis by the weighted adjacency matrix $A_{\Gamma}$.

The space $X$ is negative definite if and only if $p_{1}^{-1}+\cdots+p_{n}^{-1}-q^{-1}>0[23$, Theorem 5.2]. If it is not - implying that $q<\min \left\{p_{1}, \ldots, p_{n}\right\}$ - then we claim that $L$ is not QA. Consider the space $-Y=\Sigma(\bar{L})$. It is the boundary of plumbing on a star-shaped graph $\Gamma^{\prime}$ with $n$ rays of length 1 and one of length $q-1$ emanating from the star vertex. The vertices on rays of length 1 receive weights $-p_{1}, \ldots,-p_{n}$ in cyclic order, the vertices on the ray of length $q-1$ receive weight -2 , and the star vertex receives weight -1 . Under the assumption that $\Gamma$ is not negative definite, the graph $\Gamma^{\prime}$ is by another application of [23, Theorem 5.2].

\footnotetext{
${ }^{2}$ Michael Eisermann points out that this is direct from the presentation of $11 n 50$ as a symmetric union in Figure 1 (cf. [16, Theorem 5]).
} 
Now we appeal to some facts for which a detailed account would extend too far beyond the scope of this note. Since the star vertex has degree $\geq 3$ and weight -1 , an application of Laufer's algorithm terminates at the $0^{\text {th }}$ iteration, and shows at once that the space $-Y=Y_{\Gamma^{\prime}}$ is not the link of a rational surface singularity $[17$, Section 4]. The invariant $H F^{+}(Y)=H F^{+}\left(-Y_{\Gamma^{\prime}}\right)$ is identified with a particular $\mathbb{Z}[U]$-module $\mathbb{H}^{+}\left(\Gamma^{\prime}\right)$, as detailed in [28, Section 2]. On the other hand, Némethi has proven that if $H F^{+}\left(-Y_{\Gamma^{\prime}}\right) \cong \mathbb{H}^{+}\left(\Gamma^{\prime}\right)$ and $Y_{\Gamma^{\prime}}$ is an L-space, then $Y_{\Gamma^{\prime}}$ is the link of a rational surface singularity [22, Proposition 4.1.2]. It follows that $Y$ is not an L-space, so $L$ is not QA in this case.

Therefore, we may assume henceforth that $X$ is negative definite. Now suppose that $L$ were QA, so that $-\Sigma(L)=\partial W$, where $W$ is a negative definite 4-manifold with $H_{1}(W)=0$. Since $\operatorname{det}(L)>0$, it follows that $-\Sigma(L)$ is a rational homology sphere. We proceed as in the proof of Theorem 1.3 and analyze how $\left(H_{2}(X), Q_{X}\right)$ can embed into the lattice $-\mathbb{Z}^{n}=\left\langle E_{1}, \ldots, E_{n}\right\rangle$. To every vertex of $\Gamma$ corresponds a vector in $-\mathbb{Z}^{n}$. If two distinct vertices of weight -2 gave rise to vectors with the same reduction $(\bmod 2)$, then a change of basis of $-\mathbb{Z}^{n}$ puts these vectors in the form $E_{1}+E_{2}$ and $E_{1}-E_{2}$. These in turn correspond to a pair of columns of the matrix $A$ representing the map $H_{2}(X) /$ Tors $\rightarrow H_{2}(X \cup W) /$ Tors supported in the first two rows. The induced $2 \times 2$ minor has determinant \pm 2 , in contradiction to Lemma 2.1.

Now consider a ray of length $p_{i}-1$. Each vector corresponding to one of its vertices has weight -2 . Since no two have the same reduction $(\bmod 2)$, it follows that they can be put in the form $E_{1}^{i}-E_{2}^{i}, \ldots, E_{p_{i}-1}^{i}-E_{p_{i}}^{i}$, moving away from the star vertex. Furthermore, vectors corresponding to distinct rays are orthogonal, and again no two have the same reduction $(\bmod 2)$. It follows that all the basis vectors $E_{j}^{i}, 1 \leq i \leq n, 1 \leq j \leq p_{i}$, are distinct, and in turn that the star vertex corresponds to the vector $-E_{1}^{1}-\cdots-E_{1}^{n}$. Consider the vector $x$ corresponding to the vertex of weight $-q$. Its inner product with $-E_{1}^{1}-\cdots-E_{1}^{n}$ is non-zero, so in its expansion with respect to the chosen basis of $-\mathbb{Z}^{n}$, it has some term of the form $a \cdot E_{1}^{i}$ with $a \neq 0$. Since $x$ is orthogonal to all those vectors corresponding to the $i^{t h}$ ray of $\Gamma$, its expansion takes the form $a\left(E_{1}^{i}+\cdots+E_{p_{i}}^{i}\right)+$ (additional terms). It follows that $q \geq|a| \cdot p_{i} \geq \min \left\{p_{1}, \ldots, p_{n}\right\}$. Now suppose by way of contradiction that equality held throughout. Then in fact $x=E_{1}^{i}+\cdots+E_{p_{i}}^{i}$. Consider the rows of the matrix $A$ corresponding to the vectors $E_{1}^{i}-E_{2}^{i}, \ldots, E_{p_{i}-1}^{i}-E_{p_{i}}^{i}$, and $E_{1}^{i}+\cdots+E_{p_{i}}^{i}$. These are $p_{i}$ columns supported in $p_{i}$ rows, whose induced $p_{i} \times p_{i}$ minor has determinant $\pm p_{i} \neq \pm 1$, in contradiction to Lemma 2.1. Consequently, $q>\min \left\{p_{1}, \ldots, p_{n}\right\}$, as desired.

The converse statement in [3, Theorem 3.2(1)] completes the proof of the Proposition.

Proof of Theorem 1.4. Let $L$ denote the pretzel link appearing in the statement of the Theorem. As a Montesinos link, it is notated by $M\left(e ;\left(p_{1}, 1\right), \ldots,\left(p_{n}, 1\right),\left(q_{1},-1\right), \ldots\right.$, $\left.\left(q_{m},-1\right)\right)$.

If $e<m-1$, then $L$ has the equivalent description $M\left(0 ;\left(p_{1}, 1\right), \ldots,\left(p_{n}, 1\right),\left(q_{1}, q_{1}-\right.\right.$ $\left.1), \ldots,\left(q_{e}, q_{e}-1\right),\left(q_{e+1},-1\right), \ldots,\left(q_{m},-1\right)\right)$. If $e+n \geq 2$, then the diagram resulting from this description is adequate and non-alternating. It follows that $L$ is $\overline{K h}$-thick 
[14, Proposition 5.1] and hence not QA in this case. If $e+n \leq 1$, then either $e=1, n=0$, or $e=0, n=1$. In the first case, $m>e+1=2$, and $L$ is non-QA by applying Proposition 2.2 to $\bar{L}$. In the second case, Proposition 2.2 applies once again to $\bar{L}$ to determine when $L$ is QA (Case 3). This establishes the Theorem in case $e<m-1$.

If $e>m-1$, then $L$ has the equivalent description $M\left(e-m ;\left(p_{1}, 1\right), \ldots,\left(p_{n}, 1\right)\right.$, $\left.\left(q_{1}, q_{1}-1\right), \ldots,\left(q_{m}, q_{m}-1\right)\right)$. Its associated diagram is connected and alternating, so $L$ is QA in this case (Case 1). Also, if $e=m-1=0$, then the Theorem follows by a combination of Proposition 2.2 and [3, Theorem 3.2(2)] (Case 4).

It stands to consider the case that $e=m-1>0$ (Case 2). We prove that $L$ is QA by induction on $e+q_{1}+\cdots+q_{m}$. Consider a crossing appearing in the tassle with $-q_{m}$ half-twists. The resolution $L_{0}$ is the link $P\left(e ; p_{1}, \ldots, p_{n},-q_{1}, \ldots,-q_{m-1}\right)$, while the resolution $L_{1}$ is the link $P\left(e ; p_{1}, \ldots, p_{n},-q_{1}, \ldots,-\left(q_{m}-1\right)\right)$. Writing $\pi=$ $p_{1} \cdots p_{n} q_{1} \cdots q_{m-1}$ and $\sigma=e+p_{1}^{-1}+\cdots+p_{n}^{-1}-q_{1}^{-1}-\cdots-q_{m-1}^{-1}$, we calculate $\operatorname{det}\left(L_{0}\right)=\pi \cdot \sigma, \operatorname{det}\left(L_{1}\right)=\pi \cdot\left(q_{m}-1\right) \cdot\left(\sigma-\left(q_{m}-1\right)^{-1}\right)$, and $\operatorname{det}(L)=\pi \cdot q_{m} \cdot\left(\sigma-q_{m}^{-1}\right)$. Note in particular that the expression for each determinant is positive, since $e=m-1$ and there are at most $m$ negative terms in each sum, with each term $\geq-1 / 2$. The equality $\operatorname{det}(L)=\operatorname{det}\left(L_{0}\right)+\operatorname{det}\left(L_{1}\right)$ is immediate. Now, $L_{0}$ has the same value $e$ and one fewer negative term, so as in the case $e>m-1$ treated above, this link has a connected, alternating diagram, hence is QA. If $q_{m}>3$, then the link $L_{1}$ is QA by induction. Otherwise, $q_{m}=3$, and so $L_{1}=P\left(e ; p_{1}, \ldots, p_{n},-q_{1}, \ldots,-q_{m-1},-2\right)=$ $P\left(e-1 ; p_{1}, \ldots, p_{n},-q_{1}, \ldots,-q_{m-1}, 2\right)=P\left(e-1 ; 2, p_{1}, \ldots, p_{n},-q_{1}, \ldots,-q_{m-1}\right)$. If $e-1=m-2>0$, then $L_{1}$ is QA by induction, while if $e-1=m-2=0$, then $L_{1}$ is QA by Proposition 2.2. Thus, $L_{1}$ is QA regardless, and it follows that $L$ is QA as well. This completes the induction step.

The preceding argument carries over mutatis mutandis to the case of a pretzel link which differs from $L$ by a permutation of the parameters $p_{i}$ and $q_{j}$. This completes the proof of the Theorem.

\section{Discussion.}

3.1. Further obstructions. The main principle at work in this note is the fact that for a QA link $L$, there is naturally associated to it a smooth, negative definite 4-manifold $X_{L}$ with vanishing $H_{1}$ and boundary $\Sigma(L)$. It is therefore of interest to have on hand obstructions to a 3-manifold bounding a negative definite 4-manifold with torsion-free or vanishing $H_{1}$, and to examine more closely the topology of $X_{L}$ in the hopes of developing finer obstructions to QA-ness.

In the first direction, Ozsváth-Szabó [27, Section 9.2] have developed an obstruction which makes use of the correction terms in Heegaard Floer homology, and which was subsequently sharpened by Owens-Strle [25, Theorem 2]. For the case of $-Y=$ $\Sigma(\overline{11 n 50})$, the Owens-Strle obstruction does not rule out the possibility that this space bounds a negative definite 4-manifold with torsion-free $H_{1}$. Indeed, using the plumbing graph $\Gamma$, we can calculate the correction terms of $-Y$ according to [28, Corollary 1.5]. The largest correction term has the value $8 / 25$, which passes their obstruction since it is $>1 / 4$. Therefore, the argument given in Theorem 1.3 provides information where this obstruction does not. 


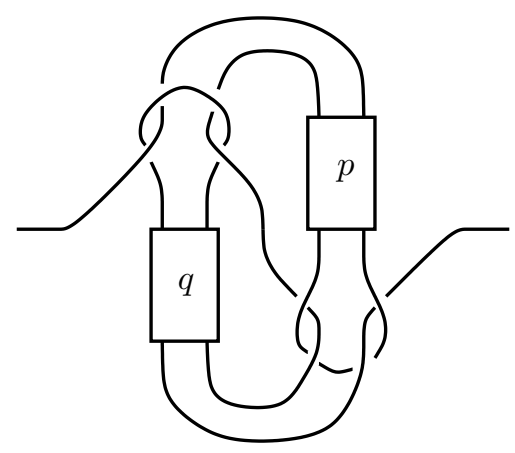

Figure 4 . The knot $K(p, q)$, split open at a point. The parameters indicate the number of right- or left-handed half-twists in the twist box, depending on whether they are positive or negative.

In the second direction, Ozsváth-Szabó have shown that $X_{L}$ is a sharp 4-manifold when $L$ is an alternating link ([30, Section 2.8], [31, Theorem 3.4]), and an early arXiv version of the paper [31] suggested that the same is true for an arbitrary QA link $L$ (math.GT/0309170, after Proposition 3.3). However, this is not the case. Indeed, $\Sigma(L)$, where $L=\overline{8_{20}}=P(3,-2,-2)$, does not bound any sharp 4-manifold [7, Proposition 7.3]. This negative result begs for an efficient means of calculating the correction terms of $\Sigma(L)$ for a QA link $L$ in general. Is it still possible to utilize $X_{L}$ in some way towards this end? What further information can we glean from $X_{L}$ to develop an obstruction to QA-ness?

3.2. Further examples. The connect-sum of $11 n 50$ with any QA link $L$ will result in a homologically thin link which is not QA. The fact that it is homologically thin follows from the behavior of the relevant knot homology groups under the connect-sum operation. The fact that it is not QA follows the proof of Theorem 1.3, noting that

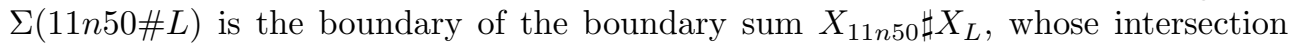
pairing decomposes as a direct sum.

Towards the end of obtaining more examples of homologically thin, non-QA hyperbolic knots, consider the following construction, due to Liam Watson, of infinitely many hyperbolic knots with the same homological invariants as the knot $11 n 50$ (in particular, determinant 25 ). The knot $11 n 50$ occurs as $K(3,0)$ in the two-parameter family of knots $K(p, q)$ depicted in Figure 4 . When the values of these parameters are even, we recover Kanenobu's two-parameter family of knots whose HOMFLY polynomial depends only on $p+q$ [12]. Allowing for arbtirary $p, q$, Watson showed that the unreduced ordinary Khovanov homology of $K(p, q)$ depends only on the value $p+q$ [35, esp. Lemma 3.1 and Section 7.4], and the same is true in the context of unreduced odd Khovanov homology by a similar argument. Since the unreduced groups are thin for $K(3,0)$, they are thin and equal for all $K(n, 3-n)$, hence the reduced versions are thin and equal as well. Furthermore, an application of the long exact sequence in knot Floer homology implies that $\widehat{H F K}(K(n, 3-n))$ is independent of $n$. A slight adaptation of Kanenobu's argument shows that these knots are hyperbolic, and the graded Floer homology groups of their branched double-covers distinguish 
these knots. Therefore, the knots $K(n, 3-n), n \geq 2$ provide the desired source of examples. Amongst them, the knot $K(2,1)=11 n 132$ is QA. However, in joint work with Watson, we show that $K(n, 3-n)$ is not QA for $|n| \gg 0$ [8].

In a different direction, work of Champanerkar-Kofman [3] and Widmer [36] indicates some progress in extending Theorem 1.4 to the more general case of Montesinos links. We expect that the methodology used herein could establish a direct analogue to Theorem 1.4 in this broader setting.

Lastly, extensive calculations by Jablan-Sazdanović [10, 11] (including corrections to some of the ones that originally appeared in [11]) indicate that amongst multicomponent links with up to 11 crossings, all except L11n77 and L11n90 are either odd-thick or non-QA. The method described here can be used to show that L11n90 is not QA, although it is odd-thin. We were unable to conclude anything further about L11n77. Therefore, it may require additional ideas to prove that it is non-QA, if indeed this is the case.

3.3. A conjecture. We close with a conjecture.

Conjecture 3.1. There exist only finitely many QA links with a given determinant.

Note the contrast between Conjecture 3.1 and the existence of infinitely many homologically thin links with the same determinant (such as the family $K(n, 3-n)$ ) cited above. In support of Conjecture 3.1, recall that there exist finitely many alternating links with a given determinant. This follows essentially because the determinant of an alternating link equals the number of spanning trees in the black graph of an alternating projection of it. Moreover, we have the following result for very small determinants.

Proposition 3.2. If $L$ is a $Q A$ link with determinant $\leq 3$, then $L$ is alternating.

Proof. Suppose that $L$ is a QA link. If $\operatorname{det}(L)=1$, then the assertion is trivial.

Next, suppose that $\operatorname{det}(L)=2$. Let $c$ denote a QA crossing in a diagram of $L$, and $L_{0}$ and $L_{1}$ the two resolutions of $L$ at $c$. Of course, both $L_{0}$ and $L_{1}$ are unknots. Let $\gamma$ denote a small unknotted arc connecting the two strands nearby the resolution in $L_{0}$, and let $K$ denote its preimage in $\Sigma\left(L_{0}\right)=S^{3}$. Then $\Sigma\left(L_{1}\right)=S^{3}$ is a non-trivial surgery on $K$; by the Dehn surgery characterization of the unknot ([15, Theorem 1.1], or [6, Theorem 2] in this special case), it follows that $K$ is the unknot. The space $\Sigma(L)$ is an integer surgery on $K$ as well, and since $\operatorname{det}(L)=2$ it follows that $\Sigma(L) \cong \mathbb{R} P^{3}$. A result of Hodgson-Rubinstein [9, Corollary 4.12] characterizes 2-bridge links as those links whose branched double-covers are lens spaces. It follows that $L$ is the Hopf link.

Lastly, suppose that $L$ is QA and $\operatorname{det}(L)=3$, and proceed as above. In this case, $L_{0}$ is the unknot, while $L_{1}$ is the Hopf link (or vice versa). Now $\Sigma\left(L_{1}\right) \cong \mathbb{R} P^{3}$ is a surgery on $K \subset S^{3}$, and the Dehn surgery characterization of the unknot once again shows that $K$ is the unknot. Hence $\Sigma(L)$ is the lens space $\pm L(3,1)$, and citing $[9$, Corollary 4.12] again shows that $L$ is a trefoil knot.

John Baldwin gives an alternative argument for the case of a determinant 3 QA link. Such a link is $\widehat{H F K}$-thin. Now using the facts that $\widehat{H F K}(L)$ is the $E_{1}$ term in a spectral sequence converging to $\widehat{H F}\left(S^{3}\right) \cong \mathbb{Z}_{(0)}$, and that $\widehat{H F K}_{d}(L, i) \cong \widehat{H F K}_{d-2 i}(L,-i)$ [29], it follows that $L$ has the knot Floer homology of a trefoil knot. Since a trefoil is uniquely determined by its knot Floer homology [5], the result follows. 
We remark that if there were infinitely many prime QA links of some fixed determinant, then amongst their branched double-covers we would obtain an infinite family of irreducible L-spaces with the same graded Heegaard Floer homology groups [8]. No such family is known as of this writing. For that matter, it remains unknown whether all the prime factors of a composite QA link are necessarily QA.

\section{Acknowledgments.}

Thanks to John Baldwin, Abhijit Champanerkar, Michael Eisermann, Slavik Jablan, and Liam Watson for helpful correspondence, and to the anonymous referee for a careful reading and thoughtful remarks.

\section{References}

[1] J. A. Baldwin, Heegaard Floer homology and genus one, one-boundary component open books, J. Topol. 1 (2008), no. 4, 963-992.

[2] J. A. Baldwin and W. D. Gillam, Computations of Heegaard-Floer knot homology, math.GT/0610167 (2007)

[3] A. Champanerkar and I. Kofman, Twisting quasi-alternating links, arXiv:0712.2590 (2009)

[4] S. K. Donaldson, The orientation of Yang-Mills moduli spaces and 4-manifold topology, J. Differential Geom. 26 (1987), no. 3, 397-428.

[5] P. Ghiggini, Knot Floer homology detects genus-one fibred knots, Amer. J. Math. 130 (2008), no. $5,1151-1169$.

[6] C. M. Gordon and J. Luecke, Knots are determined by their complements, J. Amer. Math. Soc. 2 (1989), no. 2, 371-415.

[7] J. Greene, On closed 3-braids with unknotting number one, arXiv:0902.1573 (2009)

[8] J. Greene and L. Watson, In progress.

[9] C. Hodgson and J. H. Rubinstein, Involutions and isotopies of lens spaces, in Knot theory and manifolds (Vancouver, B.C., 1983), Vol. 1144 of Lecture Notes in Math., 60-96, Springer, Berlin (1985).

[10] S. Jablan, Private communication (2009)

[11] S. Jablan and R. Sazdanović, Quasi-alternating links and odd homology: computations and conjectures, arXiv:0901.0075 (2009)

[12] T. Kanenobu, Infinitely many knots with the same polynomial invariant, Proc. Amer. Math. Soc. 97 (1986), no. 1, 158-162.

[13] A. Kawauchi, A survey of knot theory, Birkhäuser Verlag, Basel (1996), ISBN 3-7643-5124-1. Translated and revised from the 1990 Japanese original by the author.

[14] M. Khovanov, Patterns in knot cohomology. I, Experiment. Math. 12 (2003), no. 3, 365-374.

[15] P. B. Kronheimer, T. S. Mrowka, P. Ozsváth, and Z. Szabó, Monopoles and lens space surgeries, Ann. of Math. (2) 165 (2007), no. 2, 457-546.

[16] C. Lamm, Symmetric unions and ribbon knots, Osaka J. Math. 37 (2000), no. 3, 537-550.

[17] H. B. Laufer, On rational singularities, Amer. J. Math. 94 (1972) 597-608.

[18] W. B. R. Lickorish, An introduction to knot theory, Vol. 175 of Graduate Texts in Mathematics, Springer-Verlag, New York (1997), ISBN 0-387-98254-X.

[19] W. B. R. Lickorish and M. B. Thistlethwaite, Some links with nontrivial polynomials and their crossing-numbers, Comment. Math. Helv. 63 (1988), no. 4, 527-539.

[20] C. Manolescu, An unoriented skein exact triangle for knot Floer homology, Math. Res. Lett. 14 (2007), no. 5, 839-852.

[21] C. Manolescu and P. Ozsváth, On the Khovanov and knot Floer homologies of quasi-alternating links, in Proceedings of the 14th Gökova Geometry-Topology Conference, 60-81, International Press, Berlin (2007).

[22] A. Némethi, Lattice cohomology of normal surface singularities, Publ. Res. Inst. Math. Sci. 44 (2008), no. 2, 507-543. 
[23] W. D. Neumann and F. Raymond, Seifert manifolds, plumbing, $\mu$-invariant and orientation reversing maps, in Algebraic and geometric topology (Proc. Sympos., Univ. California, Santa Barbara, Calif., 1977), Vol. 664 of Lecture Notes in Math., 163-196, Springer, Berlin (1978).

[24] B. Owens and S. Strle, Rational homology spheres and the four-ball genus of knots, Adv. Math. 200 (2006), no. 1, 196-216.

$[25] \longrightarrow$, A characterisation of the $\mathbb{Z}^{n} \oplus \mathbb{Z}(\delta)$ lattice and definite nonunimodular intersection forms, arXiv:0802.1495 (2008)

[26] P. Ozsváth, J. Rasmussen, and Z. Szabó, Odd Khovanov homology, arXiv:0710.4300 (2008)

[27] P. Ozsváth and Z. Szabó, Absolutely graded Floer homologies and intersection forms for fourmanifolds with boundary, Adv. Math. 173 (2003), no. 2, 179-261.

[28] — On the Floer homology of plumbed three-manifolds, Geom. Topol. 7 (2003) 185-224 (electronic).

[29] - Holomorphic disks and knot invariants, Adv. Math. 186 (2004), no. 1, 58-116.

[30] - Knots with unknotting number one and Heegaard Floer homology, Topology 44 (2005), no. $4,705-745$

[31] - On the Heegaard Floer homology of branched double-covers, Adv. Math. 194 (2005), no. $1,1-33$

[32] J. Rasmussen, Knot polynomials and knot homologies, in Geometry and topology of manifolds, Vol. 47 of Fields Inst. Commun., 261-280, Amer. Math. Soc., Providence, RI (2005).

[33] A. Shumakovitch, KhoHo pari package, www.geometrie.ch/KhoHo/ (2009),

[34] - Patterns in odd Khovanov homology, In preparation (2009)

[35] L. Watson, Knots with identical Khovanov homology, Algebr. Geom. Topol. 7 (2007) 1389-1407.

[36] T. Widmer, Quasi-alternating Montesinos links, arXiv:0811.0270 (2008)

Department of Mathematics, Columbia University, New York, NY 10027

E-mail address: josh@math.columbia.edu 